\title{
A novel 3-base deletion (IVS3+2_4delTGG) of the hydroxymethylbilane synthase gene in a Brazilian patient with acute intermittent porphyria
}

\author{
Georgina Severo Ribeiro ${ }^{1,6}$, Paulo Eurípedes Marchiori ${ }^{2}$, Mário Hiroyuki Hirata ${ }^{3}$, Ivanise Rebecchi ${ }^{3}$, \\ Adriana Natsue Ozaki ${ }^{3}$, Maria Aparecida Nagai ${ }^{4}$, Mariana Lopes dos Santos ${ }^{4}$, \\ Raimundo Antonio Gomes Oliveira ${ }^{5}$ and Orlando Cesar de Oliveira Barretto ${ }^{6}$ \\ ${ }^{1}$ Departamento de Patologia, Faculdade de Medicina, Universidade Federal Fluminense, Rio de Janeiro, \\ RJ, Brazil. \\ ${ }^{2}$ Departamento de Neurologia, Faculdade de Medicina, Universidade de São Paulo, São Paulo, SP, Brazil. \\ ${ }^{3}$ Departamento de Análises Clínicas e Toxicológicas, Faculdade de Ciências Farmacêuticas, \\ Universidade de São Paulo, São Paulo, SP, Brazil. \\ ${ }^{4}$ Departamento de Radiologia, Faculdade de Medicina, Universidade de São Paulo, São Paulo, SP, Brazil. \\ ${ }^{5}$ Divisão de Hematologia, Universidade Federal do Maranhão, São Luis, MA, Brazil. \\ ${ }^{6}$ Departamento de Psiquiatria, Faculdade de Medicina, Universidade de São Paulo, São Paulo, SP, Brazil.
}

\begin{abstract}
Acute intermittent porphyria (AIP, OMIM 176000) is an autosomal dominant metabolic disease caused by mutations in the gene encoding hydroxymethylbilane synthase (HMBS; EC 4.3.1.8; formely named porphobilinogen deaminase, PBGD), mapped to chromosome 11q23.3. We describe a novel mutation of the HMBS gene, a de novo 3-base deletion in the splicing donor site of intron 3 (IVS3+2_4delTGG) in a woman affected by AIP. RT-PCR analysis revealed an abnormal HMBS mRNA, compatible with exon 3 skipping.
\end{abstract}

Key words: acute intermittent porphyria, hydroxymethylbilane synthase, porphobilinogen deaminase, $H M B S$ gene.

Received: September 15, 2006; Accepted: June 11, 2007.

Acute intermittent porphyria (AIP, OMIM 176000) is an autosomal dominant metabolic disease caused by partial deficiency of hydroxymethylbilane synthase (HMBS, EC.4.3.1.8; formerly known as porphobilinogen deaminase, PBGD). The enzyme is involved in the heme biosynthetic pathway, and the $H M B S$ gene is located on chromosome 11q23.3. AIP is clinically characterized by severe abdominal pain, constipation, vomiting, neurological signs and symptoms, and the characteristic dark urine. Clinical manifestations, however, are present in only $10 \%-20 \%$ of mutation carriers. The disease is more prevalent in females than in males. In women, the crisis usually occurs in the premenstrual period, suggesting the influence of female hormones.

More than 200 mutations associated with AIP, including deletions, insertions, missense, nonsense and splicing mutations, have been detected throughout the $H M B S$ gene, except in exon 2, and most of them have been limited to single

Send correspondence to Orlando Cesar de Oliveira Barretto. Departamento de Psiquiatria, Faculdade de Medicina, Universidade de São Paulo, Av. Pedroso de Morais 70, apt.101, 05420-000 São Paulo, SP, Brazil. E-mail: ocdobarr@usp.br. families (Cappelini et al., 2002). Here we describe a novel $H M B S$ splicing-site mutation in a woman affected by AIP.

The clinical diagnosis of AIP in the 24-year-old female was based on a psychomotor crisis induced by infection and emotional stress, which was characterized by tachycardia, labile arterial hypertension, and gastrointestinal symptoms such as abdominal colic and nausea. She also developed a neuropsychiatric syndrome, which included mental confusion, behavior changes, seizures and coma. Laboratory tests revealed that the urine turned dark when exposed to light and showed elevated urine porphobilinogen levels $(50.5 \mathrm{mg} / 24 \mathrm{~h}$ urine, reference normal values 1.0-1.5 mg/24 h urine). A slight decrease of erythrocyte HMBS activity (122.3 pmol uroporphyrin/hour/mg Hb at $37^{\circ} \mathrm{C}$ ) was detected (normal range 130 to $194 \mathrm{pmol}$ ). The patient's parents and sister showed HMBS activity in the normal range (Figure 1A, I-1: 180, I-2: 235, II-2: 204 pmol uroporphyrin/hour/mg $\mathrm{Hb}$ ).

Informed consent for the genetic studies was obtained from the patient and her relatives. Genomic DNA was extracted from peripheral blood leukocytes using a standard salting-out method (Salazar et al., 1998). All coding exons 
and exon-intron boundaries of the $H M B S$ gene were amplified by polymerase chain reaction (PCR) and the fragments were submitted to Single Strand Conformation Polymorphism (SSCP) analysis (Schreiber et al., 1995). DNA fragments with abnormal SSCP patterns when compared to a normal control DNA were directly sequenced, as previously described (Ribeiro et al., 2002).

In the patient, the fragment containing exon 3 and its flanking regions showed atypical banding patterns (Figure 1B). This exon encodes amino acid residues, which interact with the dipyrromethane cofactor, thus pointing to its importance for HMBS function (Shoolingin-Jordan, 1995). This abnormal fragment was submitted to direct sequencing, which disclosed a 3-base deletion (IVS3+2_4delTGG) (Figure 1D). This deletion disrupts the consensus sequence of the donor-splicing site AG/GTGGGTGCA at the exonintron junction and suggests a possible mRNA splicing abnormality. No abnormality was detected in the SSCP analysis of this fragment from the patient's parents (Figure 1B).

A
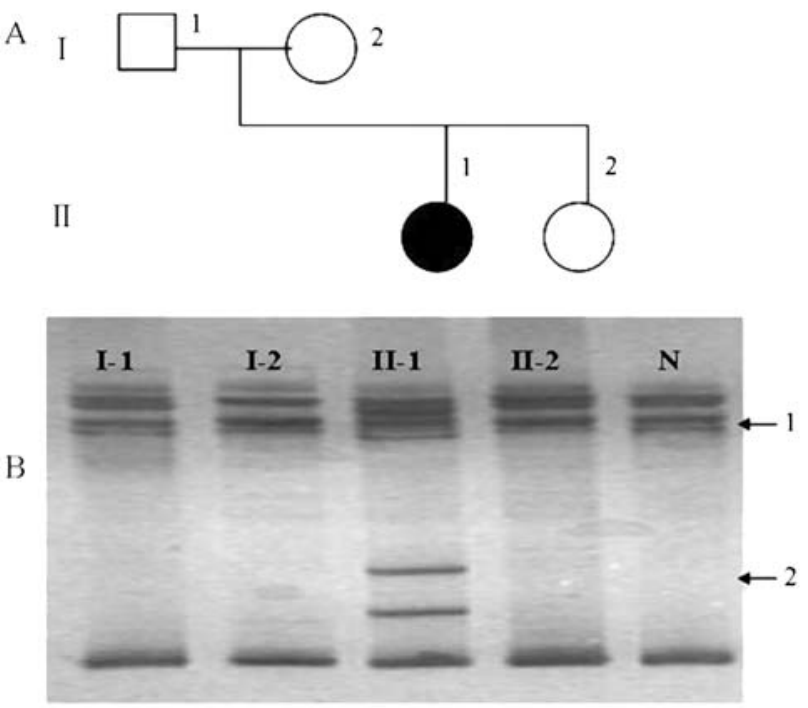

c

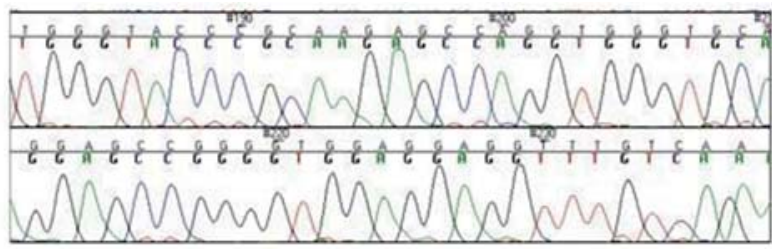

D)

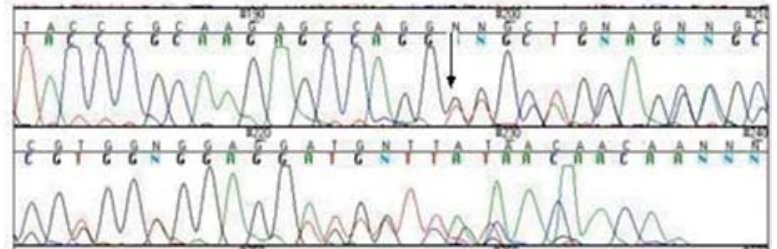

Figure 1 - A, Family pedigree. B, SSCP analysis of the exon 3/intron 3 fragment of the hydroxymethylbilane synthase gene disclosed an abnormal banding pattern in the patient (II-2). N, normal control. C, D, Electrofluorograms showing partial nucleotide sequence of the hydroxymethylbilane synthase gene in a normal control (C) and in the patient (D). The TGG deletion in the patient (arrow) disrupts a consensus sequence of the donor-splicing site G/GTGGGTGCA.
The deletion was confirmed by the amplifyication of transcription products by RT-PCR. Total RNA, obtained from peripheral blood mononuclear cells and isolated by Ficoll-Hypaque density gradient centrifugation, was reversely transcribed to cDNA using SuperScript II Rnase H-Reverse Transcriptase (Invitrogen) and random hexamers primers. Amplification of cDNA was performed by PCR with two sets of primers (Whatley et al., 1999), encoding the entire $H M B S$ cDNA sequence divided in two overlapping fragments of 842 and 740 base pairs, which included exons 1 to 12 and 9 to 15 , respectively. The presence of a smaller transcript in addition to the expected $842 \mathrm{bp}$ transcript in the patient was compatible with exon 3 skipping (Figure 2). No cDNA abnormalities were found in the patient's parents and in her sister. Polymorphic loci genotyping (D18S51, D19S253, SE-33, FGA, FESFPS, D16S539, D7S820, TPOX, F13B, D8S1179, D3S1358 and TH01; Budowle and Sprecher, 2001) of the patient and her parents resulted in a $99.9999 \%$ chance of paternity.

The mutation present in the proband was neither found in 100 normal individuals tested by SSCP screening nor in other 15 AIP patients from our laboratory. Three different mutations resulting in exon 3 skipping of the hydroxymethylbilane synthase gene have been previously described (Lundin et al., 1995; Llewellyn et al. 1996; Mustajoki et al., 1998).

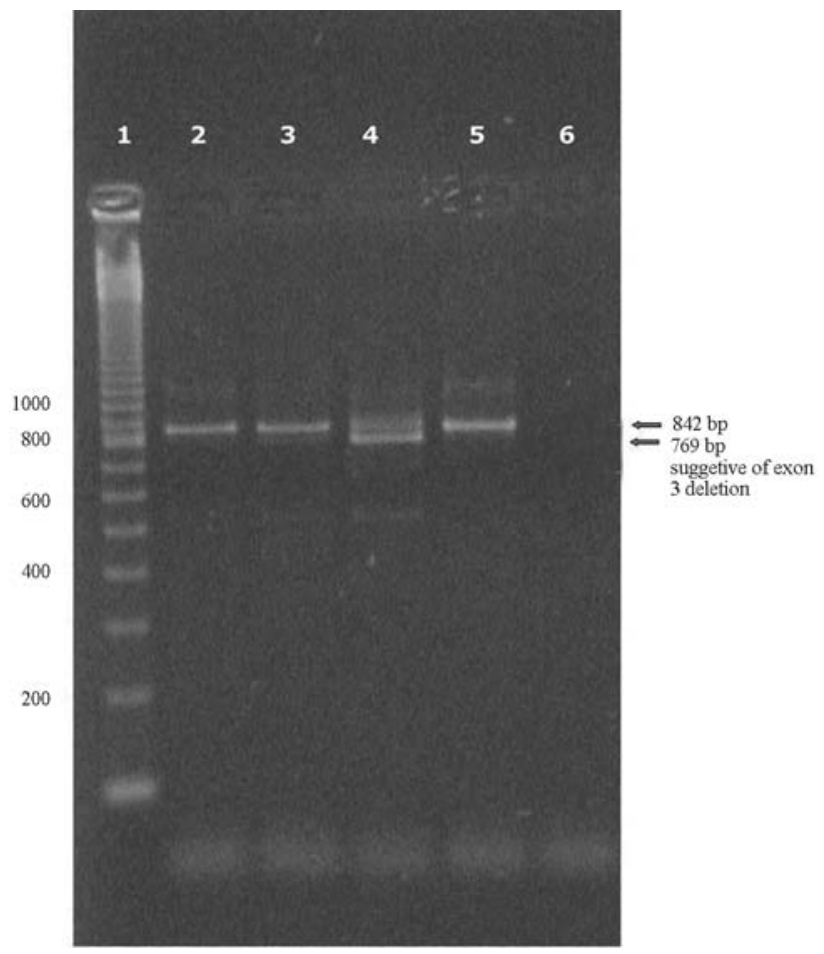

Figure 2 - cDNA analysis of exons 1 to 12 ( $842 \mathrm{bp}$ amplification product) of the hydroxymethylbilane synthase gene. Lane 1, 100 bp DNA size marker; lane 2, normal control; lane 3, patient's mother; lane 4, patient; lane 5 , patient's father; lane 6 , negative control. The smaller product detected in the patient is compatible with exon 3 skipping. 


\section{Acknowledgments}

The authors are grateful to the proband and her family. We are also indebted to Mr. Mustafa Issa for technical assistance.

\section{References}

Cappelini MD, di Montemuros FM, Di Pierro E and Fiorelli G (2002) Hematologically important mutations: Acute intermittent porphyria. Blood Cells Mol Dis 28:5-12.

Llewellyn DH, Scobie GA, Urquhart AJ, Whatley SD, Roberts AG, Harrison PR and Elder GH (1996) Acute intermittent porphyria caused by defective splicing of porphobilinogen deaminase RNA: A synonymous codon mutation at $-22 \mathrm{bp}$ from the 5' splice site causes skipping of exon 3. J Med Genet 33:437-438.

Lundin G, Hashemi J, Floderus Y, Thunell S, Sagen E, Laegreid A, Wassif W, Peters T and Anvret M (1995) Four mutations in the porphobilinogen deaminase gene in patients with acute intermittent porphyria. J Med Genet 32:979-981.

Mustajoki S, Pihlaja H, Ahola H, Petersen NE, Mustajoki P and Kauppinen R (1998) Three splicing defects, an insertion, and two missense mutations responsible for acute intermittent porphyria. Hum Genet 102:541-548.
Ribeiro GS, Marchiori PE, Puglia PMK, Nagai MA, Santos ML, Nonoyama K, Hirata MH and Barretto OC de O (2002) Porphobilinogen deaminase gene mutations in Brazilian Acute Intermittent Porphyria patients. J Clin Lab Anal 16:259-265.

Salazar LA, Hirata MH, Cavalli SS, Machado MO and Hirata RD (1998) Optimized procedure for DNA isolation from fresh and cryopreserved clotted human blood useful in clinical molecular testing. Clin Chem 44:1748-1750.

Schreiber WE, Fong F and Nassar BA (1995) Heteroduplex analysis detects frameshift and point mutations in patients with acute intermittent porphyria. Hum Genet 96:161-166.

Shoolingin-Jordan PM (1995) Porphobilinogen deaminase and uroporphyrinogen III synthase: Structure, molecular biology, and mechanism. J Bioenerg Biomemb 27:181-195.

Budowle B and Sprecher CJ (2001) Concordance study on population data base samples using the PowerPlex 16 kit andAmpFlSTR Profiler Plus kit and AmpFISTR COfiler kit. Forensic Sci 46:637-641.

Whatley SD, Woolf JR and Elder HH (1999) Comparison of complementary and genomic DNA sequencing for the detection of mutations in the HMBS gene in British patients with acute intermittent porphyria: Identification of 25 novel mutations. Hum Genet 104:505-510.

Editor: Angela M Vianna-Morgante 